\title{
On the composition of luminescence spectra from heavily doped p-type silicon under low and high excitation
}

\author{
Hieu T. Nguyen* and Daniel Macdonald \\ Research School of Engineering, College of Engineering and Computer Science, The Australian National University, \\ Canberra, ACT 2601, Australia \\ *Corresponding email address: hieu.nguyen@anu.edu.au
}

We present a systematic investigation of the effects of doping on the luminescence spectra from p-type crystalline silicon wafers. First, we explain the difference in the approaches between the line shape analysis and the generalized Planck's law in modeling the spectral shape, and connect the two methods together. After that, we elucidate the separate impacts of individual phenomena including band gap narrowing, band tails and dopant bands, Fermi level shifting, and hot carriers on the luminescence spectra in heavily-doped silicon. Finally, employing appropriate excitation levels, we can unambiguously re-examine the growing of the band tails and the broadening of the shallow dopant band, as well as the merging of the valence and shallow dopant bands together, on measured luminescence spectra.

\section{Introduction}

Due to rich features contained in photoluminescence (PL) spectra from crystalline silicon (c-Si) wafers, photoluminescence spectroscopy (PLS) has been employed to study many fundamental properties of c-Si, such as the band gap at different temperatures [1] and doping levels [2,3], absorption coefficients [4-7], doping concentrations [8], or radiative recombination rates [6,9,10]. Recently, with the advent of micro-PLS tools equipped with confocal optics, it is feasible to pinpoint to micron-scale features in c-Si wafers and solar cells to study the luminescence properties of the material and defects [11-15], or to characterize devices [16-19]. Recently, we have employed micro-PLS to qualitatively assess doping levels of localized heavily-doped regions in c-Si wafers and solar cells [20], since these regions emit a distinct luminescence peak courtesy of the bandgap narrowing effects in heavily-doped silicon at temperatures around $80 \mathrm{~K}[2,3]$. In principle, such distinct peaks could allow quantitative micron-scale PL methods for measuring doping concentrations and junction depths. However, the detailed analysis of such spectra is complicated by the fact that there are numerous physical phenomena occurring in parallel inside the heavily-doped c-Si, rather than just a simple narrowing of the band gap caused by a single effect. These phenomena can be understood by a systematic investigation of the PL spectra.

When the doping density increases, there are at least four phenomena occurring simultaneously which impact PL spectra from c-Si. Firstly, the conduction band and valence band rigidly shift towards one another due to increasing interactions among free carriers, and between free carriers and dopant atoms, causing a reduction in the band gap [21-23]. Secondly, the two band edges are perturbed and band tails are formed, yielding not only an effectively-smaller band gap but also a wider distribution of free carriers at the two band edges [24-27]. Thirdly, the shallow dopant band, located slightly above the valence band (for p-type silicon), broadens due to an increasing interaction among the dopant atoms. When the doping density approaches the Mott transition $\left(\sim 3 \times 10^{18} \mathrm{~cm}^{-3}\right)$, this dopant band slowly overlaps the valence band tail, and eventually the two bands (dopant and valence) merge together at a doping density higher than the Mott transition, forming a deeper and continuous band tail [26,28]. Fourthly, the Fermi level shifts towards the valence band (for p-type silicon) 
and eventually moves into the valence band (i.e. the semiconductor becomes degenerate) at a doping density much higher than the Mott transition [29]. The effects of these phenomena on the band diagram of c-Si are illustrated in Figure 1.

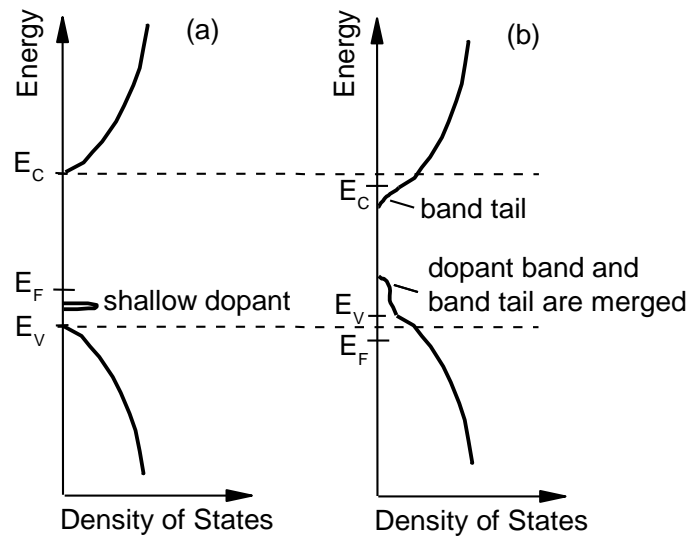

Figure 1: Illustration of the band diagram of (a) slightly-doped and (b) heavily-doped p-type c-Si. $E_{C}, E_{V}$, and $E_{F}$ are the conduction and valence band edges, and the Fermi level, respectively.

In fact, many authors presented intensive experimental results on the PL spectra from heavily-doped c-Si [2,3,30-34]. Moreover, based on the experimental PL spectra, Dumke [33], Wagner [2], and Wagner and Alamo [34] extracted the apparent band-gap shift and the position of Fermi level as a function of doping densities. However, although changes in luminescence spectra at heavy doping reflect complex combinations of the four phenomena described above, these phenomena are often lumped into two simple properties of the spectra: the energy shift of the peak, and the broadening of the width; and thus the individual effects of each phenomenon on the spectral shape are likely to be confounded [2,3,30-34].

In addition, although Schmid et al. [32] and Wagner [2] reported the PL spectra at different doping densities for $\mathrm{p}+$ silicon, the effects of the band tails and shallow dopant band, as well as their evolutions with doping densities, could not be observed in the spectra from these works. These features were either masked by the deep-level emission at low excitation levels due to unintended impurities [32], or smeared out due to the broadening of the band-to-band spectra and the relative suppression of the shallow dopant peak under high excitation levels [2,32]. Therefore, there is still a lack of empirical luminescence data, in which the effects of these phenomena are unambiguously revealed. Furthermore, although the so-called line shape analysis is commonly used to study the properties of luminescence spectra at different doping densities $[2,3,31,33]$, the formula describing the spectral shape in this method is very different from that of the generalized Planck's law [35,36], which has also been widely used to model the PL spectra [4-7,9,37-40].

In this study, we systematically re-examine the experimental data and model the PL spectra in order to separate the impacts of individual parameters on the spectral shape at both low and high excitation levels, obtained from heavily-doped p-type c-Si wafers. Moreover, in our experiments, when the shallow dopant and valence bands have not merged together, the use of a low excitation level (in this case around $4 \mathrm{~W} / \mathrm{cm}^{2}$ ) helps avoid the overlapping of the shallow dopant and band-to-band luminescence peaks. Once the two bands merge into one, the use of a high excitation level $\left(250 \mathrm{~kW} / \mathrm{cm}^{2}\right)$ can suppress the influence of unintentional deep-level centers on the PL spectra, which are evident in the low excitation spectra and greatly affect the interpretation of the PL spectral shape. First, we resolve the differences in the formulas between the line shape analysis and the 
generalized Planck's law, and connect the two methods together. We then elucidate the separate impacts of individual parameters, including the band gap, Fermi level, hot carriers, and band tails on the PL spectra from heavily-doped p-type c-Si wafers using both modeling and empirical results. The results show that, when silicon becomes degenerate, these parameters have complementary impacts on the spectral shape, and thus the energy shift of the luminescence peak cannot be simply explained by a single quantity of band-gap narrowing. Finally, we show the impacts of the band tails as well as the shallow dopant band on the PL spectra, and clarify the difference in the spectra between the high excitation (HE) and low excitation (LE) measurements. These understandings of the luminescence spectra in heavily-doped c-Si, at both low and high excitation levels, are likely to aid the further development of powerful new techniques for silicon photovoltaics based on spectral luminescence.

\section{Experimental details}

The samples investigated are float-zone boron-doped crystalline silicon (c-Si) wafers having different doping concentrations from $5.7 \times 10^{16}$ to $1.1 \times 10^{20} \mathrm{~cm}^{-3}$. Their doping densities were measured using the electrochemical capacitance-voltage (ECV) technique. In preparation for PL measurements, the wafers were chemically etched in an $\mathrm{HF} / \mathrm{HNO}_{3}$ solution to remove residual saw damage. After that, they were passivated with an 18-nm layer of $\mathrm{Al}_{2} \mathrm{O}_{3}$ deposited on both surfaces by plasma assisted atomic layer deposition (PA-ALD). They were then annealed at $450{ }^{\circ} \mathrm{C}$ in forming gas consisting of argon and hydrogen for thirty minutes to activate the surface passivation [41]. The presence of the surface passivation layers increases the excess carrier density under illumination, leading to a stronger PL signal, even for the high excitation cases.

The experimental setups of our low excitation (LE) and high excitation (HE) systems are described in detail in Ref. 7 and Ref. 15, respectively. Both systems utilize an excitation wavelength of $785 \mathrm{~nm}$ in this work. In the LE experiments, the on-sample laser spot size, on-sample excitation power, and spectral resolution are $2.2 \mathrm{~mm}$ in diameter, $150 \mathrm{~mW}$ (corresponding to an excitation intensity of $\sim 4 \mathrm{~W} / \mathrm{cm}^{2}$ ), and $5 \mathrm{~nm}$, respectively. These parameters in the HE experiments, in which the laser is coupled through a confocal microscope, are $1.7 \mu \mathrm{m}$ in diameter, $6 \mathrm{~mW}$ (corresponding to an excitation intensity of $\sim 250 \mathrm{~kW} / \mathrm{cm}^{2}$ ), and $0.25 \mathrm{~nm}$, respectively. The injection level is estimated to be in the range of $1 \times 10^{16}$ and $1 \times 10^{18} \mathrm{~cm}^{-3}$ in the LE and HE experiments, respectively. The spectral responses of both systems were determined with the same calibrated tungsten-halogen light source. The sample temperature was controlled by a liquid-nitrogen-cooled cryostat.

\section{Connections between line shape formula and generalized Planck's law}

The so-called line shape analysis has been used to describe the band-to-band luminescence spectra from a semiconductor by many authors $[2,3,31,33]$. In n-type semiconductors, the line shape formula is given by:

$\int_{0}^{\infty} f_{e}\left(E_{e}\right) D_{e}\left(E_{e}\right) f_{h}\left(E_{h}\right) D_{h}\left(E_{h}\right) d E_{e}$

where $f_{e}, D_{e}$, and $E_{e}\left(f_{h}, D_{h}\right.$, and $\left.E_{h}\right)$, respectively, are the distribution function, density of states DOS, and amount of energy above the conduction band edge $E_{C}$ (below the valence band edge $E_{V}$ ) of free electrons (holes).

There are several assumptions which need to be noted in this approach. First, the above formula only describes the ability of the material to generate photons. The emitted photon flux is also determined by the 
energy distribution of photons, which is a product of the distribution function $\left(f_{\gamma}\right)$ and DOS $\left(D_{\gamma}\right)$ of photons, and is given by [42]:

$$
f_{\lambda}(\hbar \omega) \times D_{\lambda}(\hbar \omega) \propto \frac{(\hbar \omega)^{2}}{\exp \left(\frac{\hbar \omega}{k T}\right)-1} \approx(\hbar \omega)^{2} \times \exp \left(-\frac{\hbar \omega}{k T}\right)
$$

where $\hbar \omega$ and $k T$ are the energy of emitted photons and thermal energy, respectively. Therefore, the spontaneous spectral shape of the thermal radiation from n-type semiconductors (i.e. without illumination) should be determined by the following relationship:

$$
I(\hbar \omega) \propto\left[\int_{0}^{\infty} f_{e}\left(E_{e}\right) D_{e}\left(E_{e}\right) f_{h}\left(E_{h}\right) D_{h}\left(E_{h}\right) d E_{e}\right] \times\left[(\hbar \omega)^{2} \times \exp \left(-\frac{\hbar \omega}{k T}\right)\right]
$$

However, under illumination, the Fermi level $E_{F}$ is split into two separate levels $E_{F C}=E_{F}+\Delta E_{F C}$ and $E_{F V}=E_{F}-\Delta E_{F V}$ for electrons and holes, respectively. Therefore, the distribution functions of electrons and holes under illumination $\left(f_{e, \text { illum }}\right.$ and $\left.f_{h, \text { illum }}\right)$ should be written as:

$$
f_{e, \text { illum }}\left(E_{e}\right)=\frac{1}{1+\exp \left(\frac{E_{e}-\left(E_{F}+\Delta E_{F C}\right)}{k T}\right)} \text { and } f_{h, \text { illum }}\left(E_{h}\right)=\frac{1}{1+\exp \left(\frac{\left(E_{F}-\Delta E_{F V}\right)-E_{h}}{k T}\right)} \text {. }
$$

In non-degenerate semiconductors, under low excitation levels in which the quasi-Fermi levels are far enough from the band edges, i.e. $E_{e^{-}}\left(E_{F}+\Delta E_{F C}\right)$ and $\left(E_{F}-\Delta E_{F V}\right)-E_{h}$ are several times larger than $k T$, we can rewrite $f_{e, \text { illum }}$ and $f_{h, \text { illum }}$ as:

$$
f_{e, \text { illum }}\left(E_{e}\right) \approx f_{e}\left(E_{e}\right) \times \exp \left(\frac{\Delta E_{F C}}{k T}\right) \text { and } f_{h, \text { illum }}\left(E_{h}\right) \approx f_{h}\left(E_{h}\right) \times \exp \left(\frac{\Delta E_{F V}}{k T}\right) .
$$

Therefore, under illumination, Eq. (3) should become:

$$
I(\hbar \omega) \propto\left[\int_{0}^{\infty} f_{e}\left(E_{e}\right) D_{e}\left(E_{e}\right) f_{h}\left(E_{h}\right) D_{h}\left(E_{h}\right) d E_{e}\right] \times\left[(\hbar \omega)^{2} \times \exp \left(-\frac{\hbar \omega-\left(\Delta E_{F C}+\Delta E_{F V}\right)}{k T}\right)\right]
$$

Eq. (4) is of similar form to the generalized Planck's law for non-degenerate semiconductors $[35,36]$. The first square bracket in Eq. (4) is, in principle, related to the band-to-band absorption coefficient, which is solely determined by the distribution functions and DOS of electrons and holes in the material. Some studies did not consider the energy distribution of photons $f_{\gamma} \times D_{\gamma}$, and did not account for the separation of the Fermi level under illumination, and as such their line shape formulas were different from the generalized Planck's law $[2,3,31,33]$. This difference has been noticed by Daub and Würfel [5].

However, at low temperatures, there is negligible reabsorption of the generated photons, and thus the carrier distributions do not affect the spectral shape and we can neglect the term $\Delta E_{F C}+\Delta E_{F V}$ in Eq. (4). Also, when the silicon wafers become degenerate, the excitation levels have little impact on the band-to-band PL spectral shapes, as empirically verified in Section 5 (see Figures 7e and 7f). Therefore, in principle, we can use Eq. (3) to describe the spectral shape of the band-to-band luminescence from both non-degenerate and degenerate silicon at low temperatures. For p-type silicon in this study, we simply swap the roles of electrons and holes in Eq. (3) 
together, and thus the Fermi level $E_{F}$ will be negative if it is higher than the valence band edge $E_{V}$, and positive if it moves into the valence band.

\section{Effects of different parameters on the band-to-band PL spectra}

In this section, we elucidate the separate impacts of individual parameters, including the band gap, Fermi level, and band tails on the band-to-band PL spectra from heavily-doped silicon wafers. At relatively low doping densities, for which the dopant band has a negligible impact on the PL spectra, and the DOS in both conduction and valence bands is still a parabolic function, the spectral shape at $79 \mathrm{~K}$ from silicon can be determined by Eq. (3). In addition, from Figure 2, we also have the relationship:

$\hbar \omega \pm E_{P}=E_{e}+E_{h}+E_{G}$

where $E_{P}$ and $E_{G}$ are the phonon energy and silicon band gap, respectively. '+/-' denotes the emission/absorption of phonons.

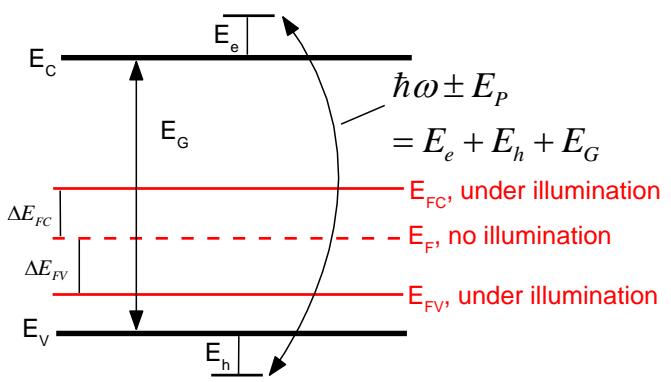

Figure 2: Illustration of the energy relationship among emitted photons $\hbar \omega$, phonons $E_{P}$ ('+' denotes a phonon emission event, whereas '-' denotes a phonon absorption event), free holes $E_{h}$, and free electrons $E_{e}$ in p-type silicon under

illumination. See the text for descriptions of the symbols.

Figure 3a compares the modeled spectrum including three components - Transverse-Acoustic (TA) phonons, Transverse-Optical (TO) phonons, and both TO and zone-center optical $\left(\mathrm{O}^{\Gamma}\right)$ phonons - with the measured LE spectrum from a lightly-doped c-Si wafer having a doping density $N_{A}$ of $5.7 \times 10^{16} \mathrm{~cm}^{-3}$ at $79 \mathrm{~K}$. The general shape of the modeled spectrum, in particular the TO component, follows the empirical spectrum closely, except for a mismatch around $1.07 \mathrm{eV}$. The peak around $1.07 \mathrm{eV}$ occurs due to free carriers recombining through neutral shallow dopant atoms, and was reported and denoted as $B_{3}^{T O}$ by Dean et al. [43]. However, in Figure 3b, the high energy side of the HE spectrum remarkably deviates from the modeling although their low energy sides match very well. The difference can be explained by the incomplete thermalization of hot carriers in the HE measurements. Under very high injection levels, more free carriers are distributed further from the band edges, and the energy distribution of the luminescence is broadened [28]. Meanwhile, this incomplete thermalization of free carriers does not change the band gap, and thus the lower energy side of the PL spectrum does not change. 


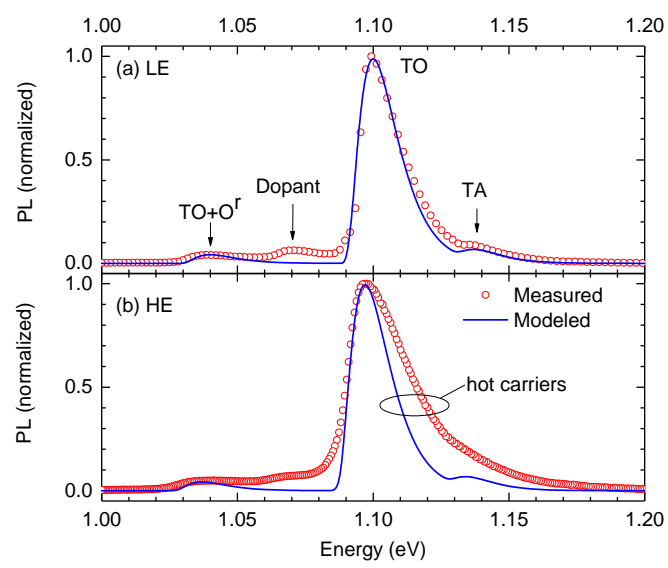

Figure 3: Comparison between measured and modeled (band-to-band, without broadening effects) PL spectra at $79 \mathrm{~K}$ under (a) LE and (b) HE levels. $N_{A}=5.7 \times 10^{16} \mathrm{~cm}^{-3}$

Near the Mott transition, there is a significant overlap between the shallow dopant and the TO components, and thus Eq. (3) does not reproduce the spectral shape well any more. When the doping density increases further, the shallow dopant band merges with the valence band and Eq. (3) becomes valid again. However, since a new deep and continuous band tail is formed due to the merging of the two bands, the spectrum is broadened significantly which is not accounted for in Eq. (3). To account for the band tails, the merging of shallow dopant band, and the incomplete ionization of free carriers, a broadening term $E_{S}$ is introduced into Eq. (3) via the relationship [2,3,33]:

$I_{\text {Broadening }}(\hbar \omega) \propto \int_{0}^{\infty} I\left(\hbar \omega^{\prime}\right) \exp \left[-\frac{\left(\hbar \omega^{\prime}-\hbar \omega\right)^{2}}{E_{S}^{2}}\right] d\left(\hbar \omega^{\prime}\right)$

In fact, the broadening model employed in Eq. (6) is a simple way to account for the band tailing effects. Above the Mott transition, the DOS in the valence band is no longer a parabolic function, but has a complex structure due to the combination of the shallow dopant band and valence band tail [26]. However, we found that Eqs. (3) and (6) are sufficient to qualitatively explain the effects of the band gap, Fermi level, and band tails on our measured PL spectra, as discussed below.

Now, we investigate the separate effects of individual parameters $\left(E_{G}, E_{F}\right.$, and $\left.E_{S}\right)$ on the PL spectra when the doping density increases. Figures $4 \mathrm{a}-\mathrm{c}$ plot the modeled PL spectra when one parameter is changed while the other two are kept the same. In Figure 4a, the narrowing of $E_{G}$ only shifts the spectra towards lower energies without altering their shapes. In Figure $4 \mathrm{~b}, E_{F}$ does not affect the spectral shape until it reaches $E_{V}$ and moves beyond $E_{V}$. The increasing $E_{F}$ (when situated in the valence band) not only shifts the spectra to higher energies, but also broadens all their components due to the band filling effects. Nevertheless, the low energy thresholds of all components (marked by broken arrows in Figure $4 \mathrm{~b}$ ) are still the same since the $E_{F}$ shifting does not change the band edge positions. In Figure $4 \mathrm{c}$, the broadening factor $E_{S}$ simply broadens the spectra, causing different components to overlap together. As a result, $E_{G}$ and $E_{F}$ have opposing effects on the spectral peak location, whereas $E_{F}$ and $E_{S}$ have similar effects on the broadening width of the PL spectra. 


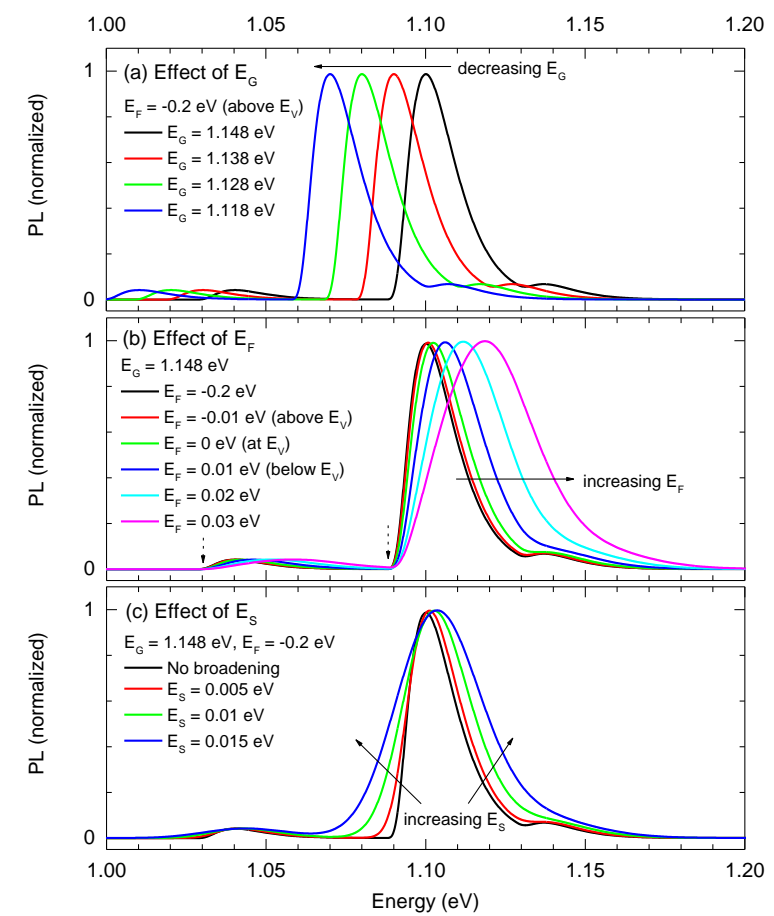

Figure 4: Modeling of individual effects of $E_{G}, E_{F}$, and $E_{S}$ on band-to-band PL spectra at $79 \mathrm{~K}$

Based on the modeling results above, one would expect that, in degenerate p-type silicon (i.e. when $E_{F}$ moves into the valence band), there is more than one single combination of $\left\{E_{G}, E_{F}, E_{S}\right\}$ giving a good fit with the measured spectra. Indeed, Figure 5 compares the HE spectra to the modeled PL spectra with various combinations of $\left\{E_{G}, E_{F}, E_{S}\right\}$. The fitted spectra, excluding the broadening parameter $E_{S}$, are also included for comparisons. The results from Figures $5 \mathrm{a}$ and $5 \mathrm{~b}$ clearly show that the choice of $\left\{E_{G}, E_{F}, E_{S}\right\}$ is arbitrary due to the complementary effects of these parameters together. These results are consistent with the results reported by Daub and Würfel [5]. By comparing the absorption curves between heavily-doped and lightly-doped silicon wafers, these authors found various amounts of apparent band-gap narrowing in the heavily-doped silicon wafers, depending on which energy range they chose to compare. Note that, the excitation level has little impact on the band-to-band spectral shape in degenerate silicon, as demonstrated in Section 5.

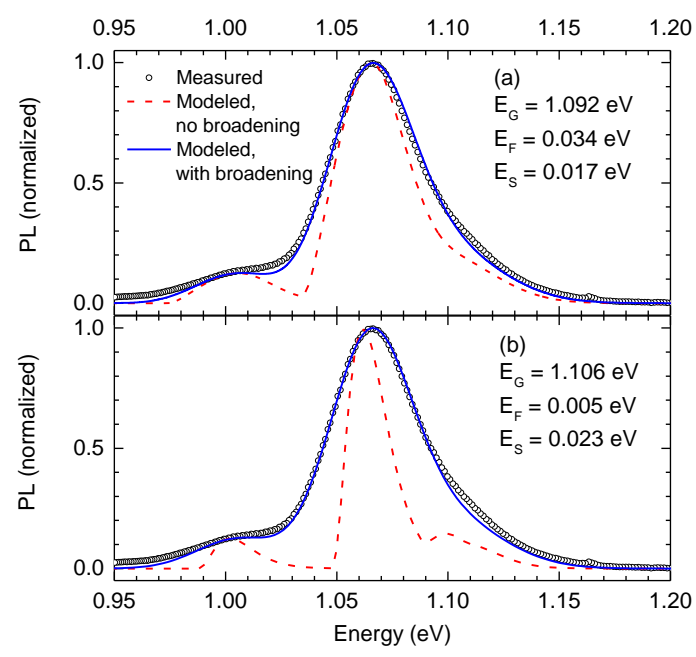

Figure 5: Measured (under HE levels) and modeled (band-to-band) PL spectra for different combinations of $E_{G}, E_{F}$, and $E_{S}$ at $79 \mathrm{~K}$ in a degenerate silicon wafer. $N_{A}=2.7 \times 10^{19} \mathrm{~cm}^{-3}$ 
However, although determining the exact values of $E_{G}$ and $E_{F}$ in degenerate silicon using Eqs. (3) and (6) has a large uncertainty, the modeling is still useful to explain the properties of the PL spectra at high doping densities. Combining the effects of $E_{G}$ and $E_{F}$, one would expect that the shifting of the spectrum peak location is smaller than the amount of band-gap narrowing in heavily-doped silicon. In addition, the spectral width should be broadened more quickly when the doping level is above the Mott transition than below the Mott transition, since both $E_{F}$ and the band tails contribute to the broadening in the former case, whereas only the band tails contribute to the broadening in the latter case. In order to fortify these predictions, we plot the HE spectra with increasing doping densities at $79 \mathrm{~K}$ in Figure 6 . When the doping density increases from $1.4 \times 10^{19}$ $\mathrm{cm}^{-3}$ to $1.1 \times 10^{20} \mathrm{~cm}^{-3}$, the spectrum peak energy is reduced only about $10 \mathrm{meV}$, whereas the amount of band-gap narrowing was reported to be between $40-60 \mathrm{meV}$ by different authors [44-46]. In addition, the full-width at half-maximum (FWHM) of the spectra increases $24 \mathrm{meV}$ when the doping density increases from $1.4 \times 10^{19} \mathrm{~cm}^{-3}$ $($ FWHM $=42 \mathrm{meV})$ to $1.1 \times 10^{20} \mathrm{~cm}^{-3}(\mathrm{FWHM}=66 \mathrm{meV})$, but increases only $12 \mathrm{meV}$ from $5.7 \times 10^{16} \mathrm{~cm}^{-3}$ $(\mathrm{FWHM}=27 \mathrm{meV})$ to $1.2 \times 10^{18} \mathrm{~cm}^{-3}(\mathrm{FWHM}=39 \mathrm{meV})$.

In fact, the much smaller shifting of the peak energy (compared to the amount of band-gap narrowing), and the rapid increment of the FWHM of the PL spectra above the Mott transition can also be observed in the experimental results reported by Wagner (see, for example, Figures 3 and 4 in Ref. 2). In our work, by modeling the separate impacts of individual parameters on the PL spectra, we can show that this modest peak energy shift is due to the opposing impacts of the band gap and the Fermi level rather than a single quantity of band-gap narrowing itself, whereas the rapid increasing FWHM is due to the combined effects of both the Fermi level and the band tails.

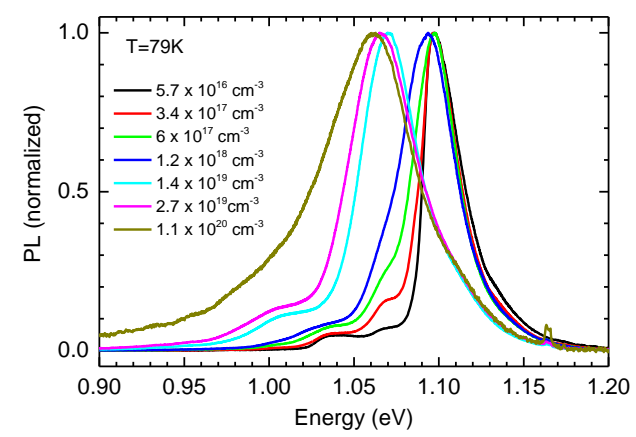

Figure 6: Evolution of measured PL spectra with doping density, under HE level at $79 \mathrm{~K}$.

\section{Evolution of PL spectra with doping densities under low and high excitations}

In this section, we examine the evolution of the PL spectra with increasing doping densities, and elucidate the difference in the spectra between the LE and HE measurements. Figure 7 shows the normalized PL spectra, under LE and HE levels at $79 \mathrm{~K}$, at different doping densities. At LE level, in Figure 7a we can observe unambiguously four peaks. Respectively, the three peaks located at 1.14, 1.1, and $1.04 \mathrm{eV}$ are the band-to-band luminescence assisted by the emissions of Transverse-Acoustic (TA) phonons, Transverse-Optical (TO) phonons, and both TO and zone-center optical $\left(\mathrm{O}^{\mathrm{F}}\right)$ phonons $[2,43]$. The peak located at $\sim 1.07 \mathrm{eV}$ is attributed to the free carriers recombining through neutral shallow dopant atoms [4,43,47]. When the dopant density increases, both the shallow dopant $(1.07 \mathrm{eV})$ and main TO $(1.1 \mathrm{eV})$ peaks broaden due to the broadening of the shallow dopant band and the extension of the band tails, and the shallow dopant peak also becomes more and more pronounced (Figures 7b-d). Above the Mott transition (Figures 7e and 7f), the shallow dopant and original 
TO peaks merge together and form a new TO peak due to the merging of the shallow dopant and valence bands. Nevertheless, the new main peak still continues broadening and moves towards lower energies with increasing doping densities. Note that, in Figure $7 \mathrm{~b}$, there is another small shoulder marked by a broken arrow at $\sim 1.09 \mathrm{eV}$. This shoulder was reported and denoted as $\mathrm{B}^{\mathrm{TO}}$ by Dean et al. [43] at lower temperatures, and was attributed to the recombination of excitons bound to the neutral shallow dopant atoms.

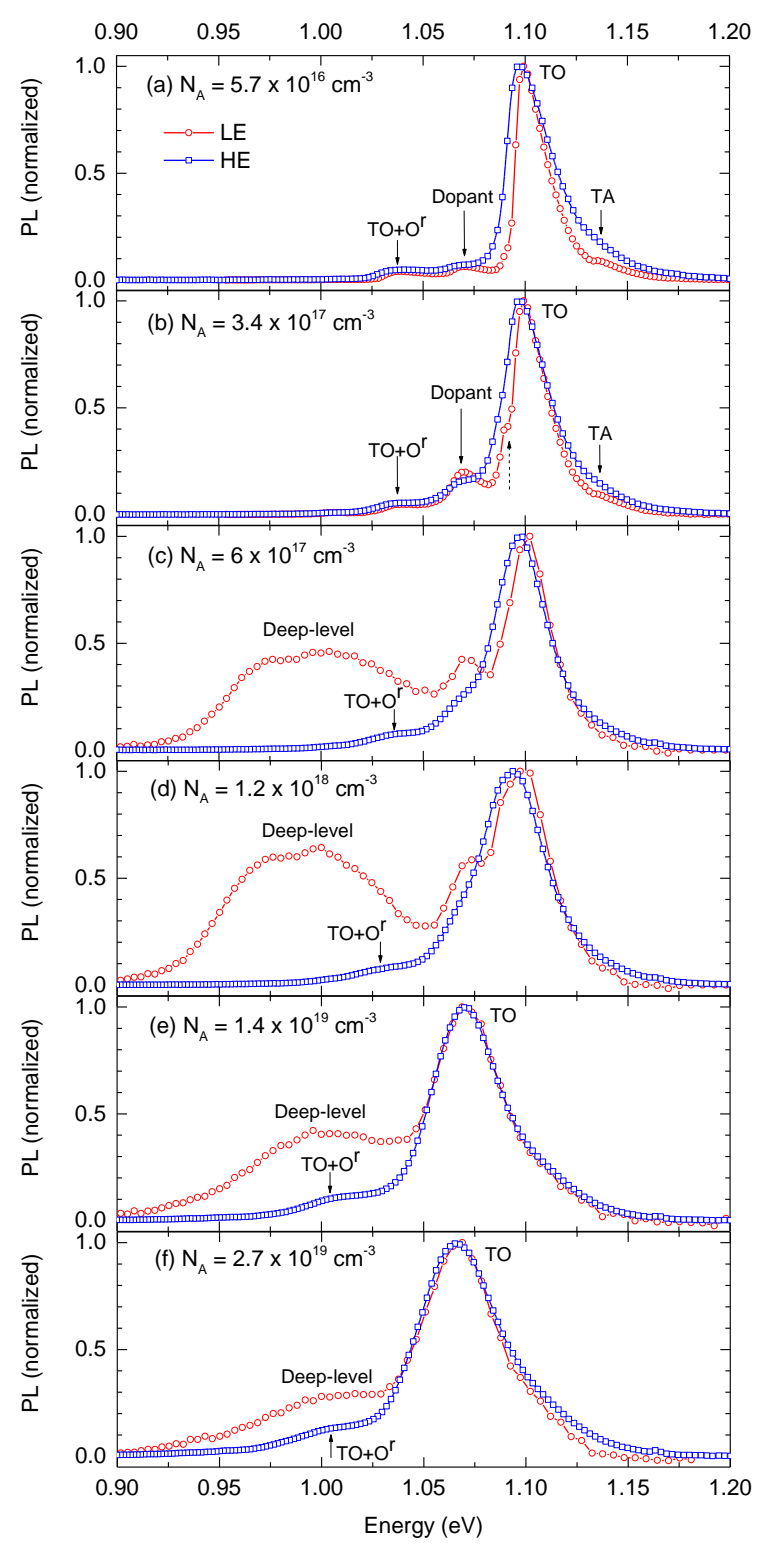

Figure 7: Comparison of normalized measured PL spectra between LE and HE measurements with different doping densities at $79 \mathrm{~K}$.

Below the Mott transition (Figures 7a-d), the spectra captured under the HE level are significantly different from those captured under the LE level. Compared to the LE spectra, the TO component of the HE spectra is broader, and their shallow dopant peak is less pronounced. The broader TO peak of the HE spectra is due to the much higher excess carrier density in the band edges, widening the energy distribution of the free carriers [28]. Meanwhile, the compression of the shallow dopant component in the HE spectra, compared to the TO component, can be explained by the weaker dependence on the excitation intensity of the shallow dopant peak. Since the shallow dopant band is located inside the forbidden gap, it can be considered as a defect band. 
Therefore, this shallow dopant peak does not increase with the excitation level as quickly as the TO peak [19]. However, when the valence and shallow dopant bands merge together, the main TO peaks of the spectra between the LE and HE measurements are similar (Figures 7e and 7f). Here, we did not try to model the PL spectra because, near the Mott transition, the band-to-band spectrum is significantly affected by the shallow dopant peak (Figures 7b-d), and as such the band-to-band spectral shape cannot be reproduced well using Eq. (3). When silicon becomes degenerate (Figures 7e and 7f), as discussed in Section 4, there is no unique solution of $\left\{E_{G}, E_{F}, E_{S}\right\}$ to describe the band-to-band spectral shape.

Surprisingly, there is another very broad peak located at $\sim 1 \mathrm{eV}$ on the LE spectra in Figures 7c-f. This peak is not present in Figure $7 \mathrm{~b}$ (boron concentration $\left.N_{A}=3.4 \times 10^{17} \mathrm{~cm}^{-3}\right)$, but then appears in Figure $7 \mathrm{c}\left(N_{A}=6 \times 10^{17}\right.$ $\mathrm{cm}^{-3}$ ) even though the doping density increases only slightly. Therefore, this broad peak is unlikely to be related to the boron dopants themselves. In addition, since the shape and intensity of this peak are consistent among different locations within the same wafer, it cannot be due to extended defects such as dislocations or oxygen/metal precipitates. Therefore, it is likely to be related to deep-level impurities introduced unintentionally but homogeneously in the wafers. Therefore, we denoted it as "deep-level" peak in Figures 7c-f. However, this "deep-level" peak completely disappears in all HE spectra. In order to verify that this "deep-level" peak is not an artifact from the LE measurements, we performed the HE measurements on the c-Si wafer having $N_{A}=$ $1.2 \times 10^{18} \mathrm{~cm}^{-3}$ with different excitation intensities. When the excitation intensity is reduced more than one order of magnitude, we start observing the "deep-level" peak on the normalized spectra (Figure 8). The results demonstrate that the "deep-level" peak is, in fact, still present on the HE spectra, but is masked by the strong luminescence signals from both the shallow dopant band and the two band edges. Furthermore, Figure 9 continues by showing the evolution of PL spectra of this silicon wafer $\left(N_{A}=1.2 \times 10^{18} \mathrm{~cm}^{-3}\right)$ when the temperature increases. The "deep-level" peak is completely suppressed at $150 \mathrm{~K}$ whereas the shallow dopant peak is still present, although the intensity of the former is stronger than the latter at $79 \mathrm{~K}$.

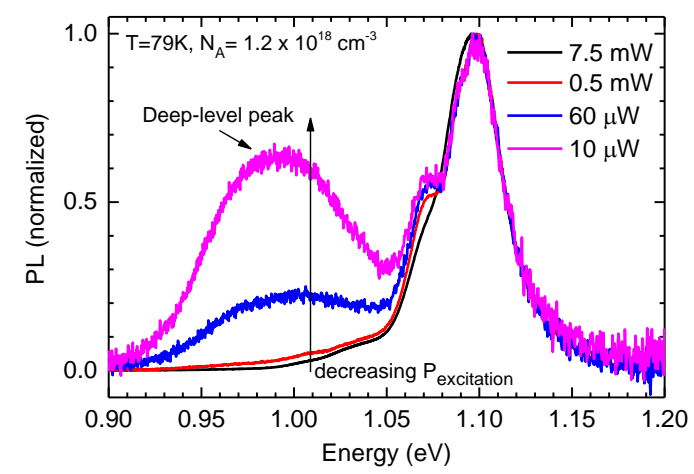

Figure 8: Normalized PL spectra, measured with the HE system at $79 \mathrm{~K}$, at different excitation powers. 


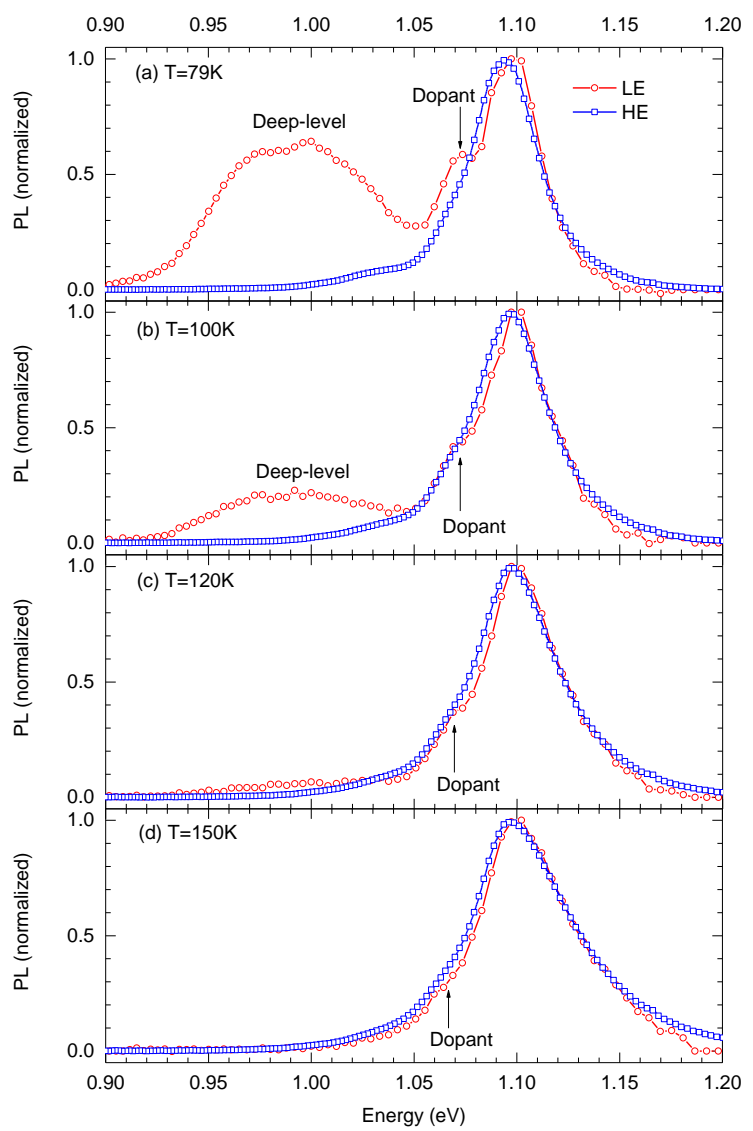

Figure 9: Comparison of normalized PL spectra between LE and HE measurements at different temperatures.

$$
N_{A}=1.2 \times 10^{18} \mathrm{~cm}^{-3} \text {. }
$$

In fact, the properties of this "deep-level" peak are similar to the findings reported by Parsons [31] and Schmid et al. [32]. These authors found a deep-level luminescence peak which was present in their lightlyexcited heavily-doped silicon wafers at low temperatures, but disappeared at either higher temperatures or high excitation levels. They attributed the observed deep-level peak to the recombination of the majority carriers in the shallow dopant band with the minority carriers, trapped by the compensating impurities introduced unintentionally into the wafers during the ingot growth. Employing the HE system, we can avoid the influence of this unexpected peak on the luminescence spectra, which otherwise greatly affects our modeling and interpretation of the PL spectra from heavily-doped c-Si.

Next, we examine the impacts of temperatures on the PL spectra of the heavily-doped silicon wafers. The LE (Figures 10a and 10b) and HE (Figure 10c) spectra show a remarkably different trend from each other. In Figure 10a, the band-to-band PL intensity increases with temperatures, contradicting the thermal quenching property of the luminescence from c-Si [6]. At low temperatures and low excitation levels, excess carriers are trapped at the "deep-level" centers, and thus the band-to-band luminescence is suppressed. However, when the temperature increases, the "deep-level" luminescence is quenched as the trapped carriers are thermally excited back to their corresponding band edges. Therefore, the excess carriers in the band edges effectively increase, leading to the increment of the band-to-band PL intensity. This increment is more than the expected thermal quenching rate of the band-to-band luminescence up to a certain temperature. However, when the temperature is high enough ( $400 \mathrm{~K}$ in this case), the thermal quenching becomes more dominant again, causing a reduction in the PL intensity as depicted in Figure 10b. 
In the HE measurements (Figure 10c), since the "deep-level” luminescence has little impact on the total spectra, the PL signal displays the expected thermal quenching property due to the known temperature dependence of the radiative recombination coefficient $[6,10]$. However, one can observe two distinct trends in the peak location of the spectra. The peak energy increases when the temperature increases from 79 to $120 \mathrm{~K}$, contradicting the expected temperature-induced band-gap narrowing in c-Si [1]. Nevertheless, with further increasing temperatures, the peak energy displays the expected reduction due to the band-gap narrowing effects. This property is observed in all heavily-doped wafers investigated in this study. One possible explanation of this behavior is that, the excess carriers in the band tails are thermally excited to higher energy levels, and thus the luminescence peak is first shifted to higher energies with increasing temperatures. Another reason could be due to the thermal broadening of the spectra, which may effectively shift the spectral peak position into slightly higher energies in a similar manner to Figure 4c. However, the HE spectra from slightly-doped silicon (not shown here) show a consistent peak shift towards low energies with increasing temperatures, thus suggest that the latter reason is unlikely to be the case. Up to a certain temperature, the amount of band-gap narrowing due to the rising temperature dominates the spectra again, and thus the PL peak shifts back to lower energies.

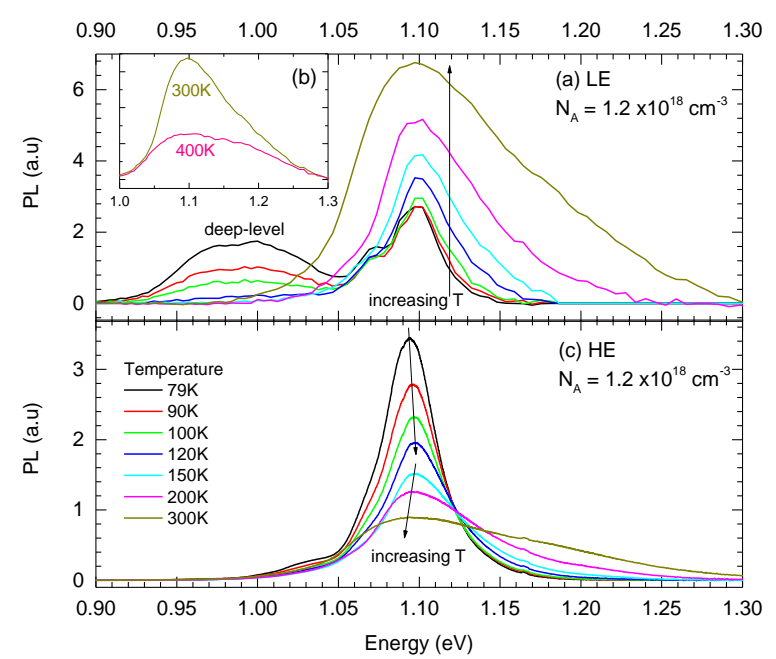

Figure 10: Temperature dependence of PL spectra, measured with both LE ( $a$ and $b$ ) and HE (c) systems. The two arrows in Figure 10c show the shifting of the peak location.

\section{Conclusion}

We have investigated the effects of doping on the photoluminescence spectra from boron-doped crystalline silicon wafers. Utilizing a low excitation level, we can avoid the overlapping of the dopant and band-to-band luminescence components. Utilizing a high excitation level from the micro-photoluminescence spectroscopy system, we can suppress the influence of the unexpected impurities on the spectra. Therefore, we can perform a systematic study on the effects of different phenomena on the luminescence spectra. We have also explained the difference between the two common approaches in studying the luminescence spectra, including the line shape analysis and generalized Planck's law.

\section{Acknowledgement}

This work has been supported by the Australian Research Council (ARC) and the Australian Renewable Energy Agency (ARENA) through research grant RND009. The Australian National Fabrication Facility is 
acknowledged for providing access to some of the facilities used in this work. The authors are in debt to Prof. H.

Tan for providing access to the spectroscopic equipment, and Dr. S. Mokkapati for assisting with some of the experimental setups.

[1] W. Bludau, A. Onton, and W. Heinke, “Temperature dependence of the band gap of silicon,” J. Appl. Phys., vol. 45, pp. 1846-1848, 1974.

[2] J. Wagner, "Photoluminescence and excitation spectroscopy in heavily doped n- and p-type silicon," Phys. Rev. B, vol. 29, pp. 2002-2009, 1984.

[3] J. Wagner, "Band-gap narrowing in heavily doped silicon at 20 and $300 \mathrm{~K}$ studied by photoluminescence," Phys. Rev. B, vol. 32, pp. 1323-1325, 1985.

[4] E. Daub and P. Würfel, "Ultralow values of the absorption coefficient of Si obtained from luminescence," Phys. Rev. Lett., vol. 74, pp. 1020-1023, 1995.

[5] E. Daub and P. Würfel, "Ultralow values of the absorption coefficient for band-band transitions in moderately doped Si obtained from luminescence," J. Appl. Phys., vol. 80, pp. 5325-5331, 1996.

[6] T. Trupke, M. A. Green, P. Würfel, P. P. Altermatt, A. Wang, J. Zhao, and R. Corkish, "Temperature dependence of the radiative recombination coefficient of intrinsic crystalline silicon,” J. Appl. Phys., vol. 94, pp. 4930-4937, 2003.

[7] H. T. Nguyen, F. E. Rougieux, B. Mitchell, and D. Macdonald, "Temperature dependence of the band-band absorption coefficient in crystalline silicon from photoluminescence,” J. Appl. Phys., vol. 115, pp. 043710-1 -043710-8, 2014.

[8] M. Tajima, "Determination of boron and phosphorus concentration in silicon by photoluminescence analysis," Appl. Phys. Lett., vol 32, pp. 719-721, 1978.

[9] P. P. Altermatt, F. Geelhaar, T. Trupke, X. Dai, A. Neisser, and E. Daub, "Injection dependence of spontaneous radiative recombination in crystalline silicon: Experimental verification and theoretical analysis," Appl. Phys. Lett., vol. 88, pp. 261901-1 -261901-3, 2006.

[10] H. T. Nguyen, S. C. Baker-Finch, and D. Macdonald, "Temperature dependence of the radiative recombination coefficient in crystalline silicon from spectral photoluminescence," Appl. Phys. Lett., vol. 104, pp. 112105-1 -112105$3,2014$.

[11] M. Tajima, Y. Iwata, F. Okayama, H. Toyota, H. Onodera, and T. Sekiguchi, "Deep-level photoluminescence due to dislocations and oxygen precipitates in multicrystalline Si," J. Appl. Phys., vol. 111, pp. 113523-1 - 113523-6, 2012.

[12] M. Tajima, "Spectroscopy and topography of deep-level luminescence in photovoltaic silicon," IEEE Journal of Photovoltatics, vol. 4, pp. 1452-1458, 2014.

[13] P. Gundel, M. C. Schubert, W. Kwapil, J. Schön, M. Reiche, H. Savin, M. Yli-Koski, J. A. Sans, G. Martinez-Criado, W. Seifert, W. Warta, and E. R. Weber, "Micro-photoluminescence spectroscopy on metal precipitates in silicon," Physica Status Solidi RRL, vol. 3, pp. 230-232, 2009.

[14] P. Gundel, M. C. Schubert, and W. Warta, "Simultaneous stress and defect luminescence study on silicon," Physica Status Solidi A, vol. 207, pp. 436-441, 2010.

[15] H. T. Nguyen, F. E. Rougieux, F. Wang, H. Tan, and D. Macdonald, "Micrometer-scale deep-level spectral photoluminescence from dislocations in multicrystalline silicon," IEEE Journal of Photovoltaics, vol. 5, pp. 799-804, 2015.

[16] R. Woehl, P. Gundel, J. Krause, K. Rühle, F. D. Heinz, M. Rauer, C. Schmiga, M. C. Schubert, W. Warta, and D. Biro, "Evaluating the aluminum-alloyed p+-layer of silicon solar cells by emitter saturation current density and optical microspectroscopy measurements,” IEEE Trans. Elec. Devices, vol. 58, pp. 441-447, 2011.

[17] P. Gundel, D. Suwito, U. Jäger, F. D. Heinz, W. Warta, and M. C. Schubert, "Comprehensive microscopic analysis of laser-induced high doping regions in silicon,” IEEE Trans. Elec. Devices, vol. 58, pp. 2874-2877, 2011.

[18] A. Roigé, J. Alvarez, J.-P. Kleider, I. Martin, R. Alcubilla, and L. F. Vega, "Microscale spatially resolved characterization of highly doped regions in laser-fired contacts for high-efficiency crystalline Si solar cells," IEEE Journal of Photovoltaics, vol. 5, pp. 545-551, 2015.

[19] H. T. Nguyen, Y. Han, M. Ernst, A. Fell, E. Franklin, and D. Macdonald, "Dislocations in laser-doped silicon detected by micro-photoluminescence spectroscopy,” Appl. Phys. Lett., vol. 107, pp. 022101-1 - 022101-5, 2015.

[20] H. T. Nguyen, D. Yan, F. Wang, P. Zheng, Y. Han, and D. Macdonald, "Micro-photoluminescence spectroscopy on heavily-doped layers of silicon solar cells," Physica Status Solidi RRL, vol. 9, pp. 230 - 235, 2015.

[21] J. C. Inkson, "The effect of electron interaction on the band gap of extrinsic semiconductors," J. Phys. C: Solid State Phys., vol. 9, pp. 1177-1183, 1976.

[22] G. D. Mahan, "Energy gap in Si and Ge: Impurity dependence,” J. Appl. Phys., vol. 51, pp. 2634-2646, 1980.

[23] K.-F. Berggren and B. E. Sernelius, "Band-gap narrowing in heavily doped many-valley semiconductors," Phys. Rev. B, vol. 24, pp. 1971-1986, 1981.

[24] M. Eswaran, B. Bergersen, J.A. Rostworowski, and R. R. Parsons, "Impurity band states in Si(P)," Solid State Communications, vol. 20, pp. 811-813, 1976.

[25] R. R. Parsons, "Photoluminescence in heavily-doped Si(P)," Can. J. Phys., vol. 56, pp. 814-826, 1978.

[26] J. R. Lowney, "Impurity bands and band tailing in moderately doped silicon,” J. Appl. Phys., vol. 59, pp. 2048-2053, 1986.

[27] P. V. Mieghem, "Theory of band tails in heavily doped semiconductors," Rev. Mod. Phys., vol. 64, pp. 755-793, 1992.

[28] P. P. Altermatt, A. Schenk, and G. Heiser, "A simulation model for the density of states and for incomplete ionization in crystalline silicon. I. Establishing the model in Si:P,” J. Appl. Phys., vol. 100, pp. 113714-1 -113714-10, 2006.

[29] K. Morigaki and F. Yonezawa, "Metal-nonmetal transition in doped semiconductors," Supplement of the Progress of Theoretical Physics, No. 57, pp. 146-155, 1975. 
[30] R. E. Halliwell and R. R. Parsons, "Photoluminescent studies of the condensed phase in phosphorus-doped silicon," Solid State Communications, vol. 13, pp. 1245-1248, 1973.

[31] R. R. Parsons, "On the origin of photoluminescence in heavily-doped silicon," Solid State Communications, vol. 29, pp. 763-766, 1979.

[32] P. E. Schmid, M. L. W. Thewalt, and W. P. Dumke, "Photoluminescence in heavily doped Si:B and Si:As," Solid State Communications, vol. 38, pp. 1091-1093, 1981.

[33] W. P. Dumke, "Comparison of band-gap shrinkage observed in luminescence from n+-si with that from transport and optical absorption measurements," Appl. Phys. Lett., vol. 42, pp. 196-198, 1983.

[34] J. Wagner and J. A. del Alamo, "Band-gap narrowing in heavily doped silicon: A comparison of optical and electrical data," J. Appl. Phys., vol. 63, pp. 425-429, 1988.

[35] P. Würfel ,"The chemical potential of radiation,” J. Phys. C: Solid State Phys., vol. 15, pp. 3967-3985, 1982.

[36] P. Würfel, S. Finkbeiner, and E. Daub, "Generalized Planck's radiation law for luminescence via indirect transitions," Appl. Phys. A, vol. 60, pp. 67-70, 1995.

[37] T. Trupke, E. Daub, and P. Würfel, "Absorptivity of silicon solar cells obtained from luminescence," Solar Energy Materials and Solar Cells, vol. 53, pp. 103-114, 1998.

[38] T. Trupke, "Influence of photon reabsorption on quasi-steady-state photoluminescence measurements on crystalline silicon,” J. Appl. Phys., vol. 100, pp. 063531-1 -063531-8, 2006.

[39] M. A. Green, "Analytical expressions for spectral composition of band photoluminescence from silicon wafers and bricks," Appl. Phys. Lett., vol. 99, pp. 131112-1 -131112-3, 2011.

[40] C. Schinke, D. Hinken, J. Schmidt, K. Bothe, and R. Brendel, "Modeling the spectral luminescence emission of silicon solar cells and wafers," IEEE Journal of Photovoltatics, vol. 3, pp. 1038-1052, 2013.

[41] W. Liang, K. J. Weber, D. Suh, S. P. Phang, J. Yu, A. K. McAuley, and B. R. Legg, "Surface passivation of borondiffused p-type silicon surfaces with $\left(\begin{array}{lll}1 & 0 & 0\end{array}\right)$ and $\left(\begin{array}{lll}1 & 1 & 1\end{array}\right)$ orientations by ALD $\mathrm{Al}_{2} \mathrm{O}_{3}$ layers," IEEE Journal of Photovoltaics, vol. 3, pp. $678-683,2013$.

[42] P. Würfel, Physics of Solar Cells: From Basic Principles to Advanced Concepts, p. 14, Wiley-VCH, 2009.

[43] P. J. Dean, J. R. Haynes, and W. F. Flood, "New radiative recombination processes involving neutral donors and acceptors in silicon and germanium,” Physical Review, vol. 161, pp. 711-729, 1967.

[44] W. P. Dumke, "Band-gap narrowing from luminescence in p-type Si," J. Appl. Phys., vol. 54, pp. 3200-3202, 1983.

[45] J. Wagner and J. A. del Alamo, "Band-gap narrowing in heavily doped silicon: A comparison of optical and electrical data," J. Appl. Phys., vol. 63, pp. 425-429, 1988.

[46] D. Yan and A. Cuevas, "Empirical determination of the energy band gap narrowing in p+ silicon heavily doped with boron," J. Appl. Phys., vol. 116, pp. 194505-1 - 194505-7, 2014

[47] B. Bergersen, J. A. Rostworowski, M. Eswaran, and R. R. Parsons, "Electron-hole droplets and impurity band states in heavily doped Si(P): Photoluminescence experiments and theory," Phys. Rev. B, vol. 14, pp. 1633-1648, 1976. 